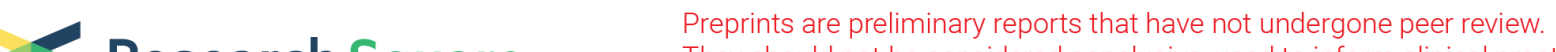 $\begin{array}{ll}\text { Research Square } & \text { They should not be considered conclusive, used to inform clinical practice, } \\ \text { or referenced by the media as validated information. }\end{array}$
}

\section{A New Prognostic and Therapeutic Immune-related Risk Signature of Colorectal Cancer}

\section{Yuan Li}

Third Affiliated Hospital of Soochow University, Changzhou 213003, China

\section{Hao Huang}

Third Affiliated Hospital of Soochow University, Changzhou 213003, China

\section{Jun Feng}

Third Affiliated Hospital of Soochow University, Changzhou 213003, China

\section{Yulan Zhu}

Third Affiliated Hospital of Soochow University, Changzhou 213003, China

\section{Tianwei Jiang}

Third Affiliated Hospital of Soochow University, Changzhou 213003, China

\section{Bin Xu}

Third Affiliated Hospital of Soochow University, Changzhou 213003, China

\section{Xiao Zheng}

Third Affiliated Hospital of Soochow University, Changzhou 213003, China

\section{Lujun Chen ( $\nabla$ clj_sz@163.com )}

Third Affiliated Hospital of Soochow University, Changzhou 213003, China

\section{Research Article}

Keywords: Colorectal cancer (CRC), microsatellite stability (MSS), immune-related genes (IRGs), immune risk signature

Posted Date: December 14th, 2021

DOI: https://doi.org/10.21203/rs.3.rs-1157680/v1

License: (c) (1) This work is licensed under a Creative Commons Attribution 4.0 International License. Read Full License 


\section{Abstract}

\section{Background}

Although some advanced colorectal cancer (CRC) patients could select immunotherapy, but still most microsatellite stability (MSS) CRC patients did not respond. Our present study aims to set up a novel system for prognostic prediction and immunotherapeutic responsiveness for MSS CRC patients.

\section{Methods}

Univariable Cox regression survival analysis and least absolute shrinkage and selector operation (LASSO) regression analysis were performed to identify prognostic genes and establish immune risk signatures. Multivariate Cox regression analysis was performed to verify whether these clinical features could predict prognosis. R package was used to analyze the relationship between the immune-related risk model and these immune cells, effector molecules, and immune checkpoints.

\section{Results}

We constructed an immune-related signature and verified its predictive capability. Immune-related signature included 12 differentially expressed IRGs (12 DE IR MSSGs), including CXCL1, CD36, FABP4, MS4A2, NRG1, VGF, GRP, HDC, XCL1, NGF, MAGEA1, and IL13. The signature consisting of 12 DE IR MSSGs was an independent and effective prognostic factor for the overall survival of CRC patients. In addition, the signature consisting of 12 DE IR MSSGs reflected the infiltration characteristics of different immunocytes in tumor immune microenvironment. The signature consisting of 12 DE IR MSSGs also had a significant correlation with immune checkpoint molecules.

\section{Introduction}

Colorectal cancer (CRC) is a major cause of morbidity and mortality of cancers over the world (1). For the patients with early stage, surgery is usually selected as the primary treatment, while those patients initially diagnosed at advanced stage with distant metastasis usually remained poor survival due to limited therapeutic strategies $(2,3)$. Thus, it's critical that the prognosis of CRC patients is significantly associated with the timing of diagnosis, and it's important to develop novel bio-markers or more accurate systems for the diagnosis and prognostic prediction.

Recently, the immune checkpoint inhibitors (ICB) therapy has been considered as a critical approach for the treatment of many tumors $(4,5)$. Microsatellite instability (MSI) is routinely caused by mutations in DNA mismatch repair (MMR) genes. In CRC, only patients with a damaged DNA mismatch repair gene and/or microsatellite instability-high (MSI-H/dMMR) seem to be sensitive to ICB (6). Microsatellites are DNA sequences (coding or non-coding sequences) with repetitive structures distributed in the genome (7). They are prone to present coding mistakes in the process of DNA replication. MMR acts to repair the potentially mutagenic misincorporation errors that occur during normal DNA replication and the absence 
of MMR results in increased rates of accumulating mutations. MSI is a marker of deficiency of DNA MMR activity (8). This mechanism leads to higher mutation rates and increased tumor antigen load. Moreover, as an important biological feature, more infiltrating immune cells and higher level of type I interferon expression were found in the tumor microenvironment of the MSI-H subtype patients, which is essential for the involvement of ICB (9-12). Unfortunatly, MSI-H/MMR-deficiency only accounts for approximately $15 \%$ of CRC cases $(13,14)$. Therefore, reliable predictive biomarkers are urgently required to monitor tumor progression and immunotherapy efficacy in MSS patients. Immune-related genes (IRGs) and tumor immune microenvironment (TIME) have become promising biomarkers for evaluating the survival of multiple cancers (15-17). Novel biomarkers can enhance the risk stratification of screening, and identify susceptibility or initial stages of the disease. Besides, specific biomarkers can determine whether a personalized treatment is required (18).

CRC patients' data and transcriptome RNA sequence data were downloaded and analyzed from The Cancer Genome Atlas (TCGA) for analysis. We estimated the MSS-mediated immune risk pattern from CRC patients and correlated it with clinical and pathological features of CRC patients. Through comprehensive genomic data analysis, we established a new MSS-mediated immune-related risk model from CRC patients. We found that 12 DE IR MSSGs were powerful prognostic biomarkers and predictors in response to immune checkpoint inhibitors. We also explored immune cells, effector molecules, and mutation data related to risk signature, which might provide novel ideas for further immunological studies and more precise immunotherapy for MSS CRC patients. Our further study aims to determine which biomarkers can determine the best predictive index for patients and can be used in personalized treatment.

\section{Results}

\section{Differential expression of DE IR MSSGs in CRC patients}

According to the TCGA dataset of 222 CRC patients, we used the R package to adjust $(P<0.05$ and I $\log 2$ (multiple changes) $\mid \geq 1.0$ ), and 21,995 DEGs were obtained. We analyzed the difference and obtained the DEGs by heat map and volcano map (Figure 1,2A). The intersection of MSS-related genes, IRGs, and DEGs in CRC identified 253 differentially expressed IRGs (DE IR MSSGs) (Figure 2B). We extracted DE IR MSSGs using the R package by enrichment analysis. The most prosperous positive terms of $\mathrm{GO}$ were as follows: "positive regulation of cytokine production", "positive regulation of peptidyl-tyrosine phosphorylation", "regulation of peptidyl-tyrosine phosphorylation", "peptidyl-tyrosine phosphorylation", "peptidyl-tyrosine modification" (Figure 2C). The most prosperous negative terms of GO were as follows: "leukocyte migration", "positive regulation of response to external stimulus", "regulation of lymphocyte activation", "leukocyte proliferation", "lymphocyte proliferation"(Figure 2D). The positive top 5 approaches obtained through KEGG analysis were as follows: "cytokine-cytokine receptor interaction”, " PI3K-Akt signaling pathway”, "MAPK signaling pathway”, "Rheumatoid arthritis”, "IL-17 signaling pathway" (Figure 2E). The negative top 5 approaches obtained through KEGG analysis were as follows: "Cytokine-cytokine receptor interaction", "Neuroactive ligand-receptor interaction”, "PI3K-Akt 
signaling pathway", "Hematopoietic cell lineage”, "JAK-STAT signaling pathway" (Figure 2F). These differential genes might play an important role in regulating these pathways.

\section{MSS-mediated development of immune-related risk characteristics in CRC patients}

Univariate Cox regression analysis was performed to study the prognostic value of these $253 \mathrm{DE}$ IR MSSGs. We identified 20 DE IR MSSGs that were significantly correlated with the OS $(P<0.05)$. Using profiles of DE IR MSSGs associated with OS, we performed LASSO regression analysis and identified 12 CRC-specific prognostic DE IR MSSGs (Figure 3). To obtain a clinically applicable risk assessment model, we established immune characteristics based on the expressions of the 12 DE IR MSSGs and their corresponding coefficients obtained from multivariate Cox regression analysis (Figure 3 ). The formula was listed as follows: risk score $=\left(-0.01640918^{\star} \mathrm{CXCL} 1\right)+\left(0.009410274{ }^{*} \mathrm{CD} 36\right)+(0.020934107 * \mathrm{FABP} 4)+$ $(-0.073249101 * \mathrm{MS} 4 \mathrm{~A} 2)+(-0.026759138 * \mathrm{NRG} 1)+(0.05610912 * \mathrm{VGF})+(-0.003939922 * \mathrm{GRP})+$ $(-0.027542663 * \mathrm{HDC})+\left(0.027187195^{*} \mathrm{XCL} 1\right)+(0.046561706 * \mathrm{NGF})+(0.044794432 * \mathrm{MAGEA} 1)+$ $(0.020605364 * \mathrm{IL} 13)+0.257498710$. We used $\mathrm{R}$ package to calculate the critical value score $=0.205878419$ of the best risk score. The patient with a score $>0.205878419$ was assigned to the high-risk group $(n=101)$, and the the patients with a score $\leq 0.205878419$ was assigned to the low-risk group $(\mathrm{n}=121)$. Table 1 lists the details of the 12 DE IR MSSGs.

\section{Clinical characteristics and OS-associated immune-related risk genes in CRC patients}

We then studied the relationship between clinical features (age, sex, clinical stage, histological grade, and TNM staging status) and risk score, which was shown in Table 2. The results showed that the risk score was significantly correlated with gender $(P<0.01)$, and other clinical features were not statistically significant. By analyzing the relationship between 12 DE IR MSSGs and OS, we found that OS was significantly better in the low-risk group (Figure $4 A, P<0.0001$ ), and the AUC was 0.738 in the OS prediction (Figure $4 B$ ). We also analyzed the survival curves of the 12 above-mentioned genes. The results showed that the higher expressions of CXCL1, MS4A2, HDC, NRG1, and IL13 indicated a better survival of CRC patients. However, the results of CD36, FABP4, VGF, GRP, XCL1, NGF, and MAGEA1 showed the opposite trend (Figure $4 C$ ). In the multivariate Cox regression model, after other clinical characteristics were adjusted, the risk score, age, sex, and clinical stage were significantly correlated with the patient's survival (Table 3). Therefore, risk groups based on risk characteristics of 12 DE IR MSSGs were independent and effective prognostic factors.

\section{Expression stability of immune-related risk genes in CRC patients}

The expressions of CXCL1, VGF, GRP, MAGEA1, and IL13 in CRC patients were significantly up-regulated compared with the normal controls, while the expressions of CD36, FABP4, MS4A2, NRG1, HDC, XCL1, and NGF were significantly down-regulated (Figure 5). Therefore, the immune-related risk model established in our present study presented as sufficient stability and feasibility.

Tumor-infiltrating immune cells are associated with risk characteristics in CRC patients 
Next, we identified the relationship between the risk characteristics of 12 DE IR MSSGs and tumorinfiltrating immune cells. The results showed that the infiltration of T cells, cytotoxic lymphocytes, B lineage, NK cells, monocytic lineage, myeloid dendritic cells, and neutrophils was higher in the low-risk group, which might be related to a better anti-tumor effect (Figure $6 A, P<0.05$ ). Moreover, 12 DE IR MSSGs were significantly correlated with T cells, cytotoxic lymphocytes, B lineage, NK cells, monocytic lineage, myeloid dendritic cells, and neutrophils (Figure 6B). Besides, we studied the association between the risk characteristics of CRC patients and immune cell infiltration to consider whether the risk score partially reflected the effect achieved. We found that the risk score was negatively correlated with the expressions of T cells, cytotoxic lymphocytes, B lineage, NK cells, monocytic lineage, myeloid dendritic cells, and neutrophils (Figure $6 C, P<0.05$ ). The results showed that the risk characteristics of 12 DE IR MSGSs were significantly correlated with tumor-infiltrating immune cells.

Relationship between immune checkpoints and immune-related risk characteristics in CRC patients

To determine the relationship between the risk characteristics of 12 DE IR MSSGs and immune checkpoint molecules, we identified differences in the expressions of immune checkpoint molecules from TCGA-COAD-READ samples between the two risk groups. In the high-risk group, the expressions of PDCD1, CTLA4, HAVCR2, LAG3, TIGIT, CD274, and PDCDLG2 were up-regulated (Figure 7A). Moreover, the risk signals of 12 DE IR MSSGs were well correlated with seven immune checkpoint molecules (Figure $7 B$ ). Our data indicated that XCL1 was positively correlated with PDCD1 and LAG3. HDC and M4AS2 were positively correlated with CTLA4 and TIGIT. There was a positive correlation between HDC and CD274. CD36 and HDC were positively correlated with HAVCR2. CD36, M4AS2, and HDC were positively correlated with PDCD1LG2 (Figure $7 C$ ). Our results showed that the risk signals of 12 DE IR MSSGs were well correlated with the expressions of immune checkpoint molecules. These molecules might be used as immune card control point treatment indicators to achieve better efficacy.

\section{Relationship between immune-related danger signals and mutations in CRC patients}

We analyzed the available somatic mutation data to evaluate the relationship between mutations and 12 DE IR MSSGs. By analyzing the relationship between the gene expression and mutation of the two groups, we found that there was no significant difference in gene mutation (Figure $8 A, B$ ). Our results showed that there were almost no differences in the mutations of these genes between the two risk groups, indicating that these genes were not changed during the mutation process, which could solve the problem of resistance of immune checkpoint inhibitor therapy and play a more significant role in the efficacy of immune checkpoint.

\section{Discussion}

Because of the tumor heterogeneity and tumor microenvironment immunosuppression of CRC, it is generally impossible to accurately evaluate the clinical results and responsiveness of ICB therapy by using a single biomarker (19). It is well known that the tumor immune microenvironment (TIME) plays a crucial role in tumor progression and occurrence, suggesting that we can try to explore immune-related 
markers for the prognositic prediction of CRC patients. It is essential to reveal the relationship between these genomic changes and the status of TIME. According to specific genomic characteristics, CRC could be genetically classified into MSI and MSS subtypes (20). MSI CRC has a better responsiveness to ICB therapy, while MSS CRC is more effective in 5-fluorouracil treatment. As for the differences in prognosis and therapy between MSI-H and MSS CRC, more studies are required to dissect the differences in biological pathways and regulation between these two subtypes of CRC (21). Understanding the relevant immune genes involved in the MSS regulation can help us find appropriate methods and provide a reliable reference for the diagnosis and treatment of immunotherapy-unresponsive patients.

Therefore, through the collation and analysis of DEGs, IRGs, and MSSs, we constructed an immunerelated risk characteristic model composed of 12 prognostic DE IR MSSs. Our results showed that CRC patients with high expressions of CXCL1, MS4A2, HDC, NRG1, and IL13 had a better OS. A number of recent studies found that CXCL1, MS4A, NRG1 and IL13 were highly expressed in a variety of tumors (2227). These results are not consistent with our research, and thus their unknown mechanism in CRC needs to be further explored. Our present results showed that CRC patients with lower expressions of CD36, FABP4, VGF, GRP, XCL1, NGF, and MAGEA1 had a better OS. Other studies confirm our conclusions, high expression of FABP4, VGF, GRP, XCL1, NGF, and MAGEA1 promoted tumor progression, and inhibition of their expression could play an anti-tumor role (28-37). However, the function of CD36 in previous studies is contrary to our results. Fang et al. have indicated that CD36 inhibits gpc4-catenin/c-myc signal transduction by promoting the expression of proteasome-dependent $\beta$, thus inhibiting downstream aerobic glycolysis and CRC (38). These molecules bring a lot of inspiration for our future research and may reference the diagnosis and treatment of CRC patients. In addition, our study found that there were significant differences in OS between different risk groups, and the survival was better in the low-risk group. Further analysis showed that 12 DE IR MSSGs were an independent prognostic factor of CRC, suggesting that 12 DE IR MSSGs could be used as a reliable prognostic tool. We noted that the risk signals of 12 DE IR MSSGs were well correlated with the expressions of immune checkpoint molecules. Surprisingly, this finding is different from previous other studies. Not all patients with MSS colorectal cancer are insensitive to immune checkpoint inhibitors. Based on our subgroup analysis, we found higher expression of PD-1/PD-L1, CTLA-4, TIM3, LAG3, TIGIT and PD-L2 in the high-risk group. Patients in high-risk groups may respond to these immune checkpoint treatments, and this model may be a useful predictor of immune checkpoint therapy. We conducted mutation analysis, and the mutations of these genes were almost not different between the two risk groups, indicating that these genes were not changed during the mutation process, which could solve the problem of resistance of immune checkpoint inhibitor therapy and play a more significant role in the efficacy of immune checkpoint.

The prognostic model consisting of 12 DE IR MSSs had a strong predictive power for the prognosis of CRC patients, which might help predict patients' response to immunotherapy and guide more effective immunotherapy strategies. Next, we will collect clinical samples for further verification to confirm the accuracy and applicability of the model. We hope that this model might provide more novel and in-depth insights into the development of new immunotherapy and offer a comprehensive understanding and a unique perspective for the prognositic evaluation of CRC patients. 


\section{Conclusion}

We constructed and verified a new, immune-related prognostic signature associated with MSS CRC patients. This model might provide more novel and in-depth insights into the development of new immunotherapy and offer a comprehensive understanding and a unique CRC perspective.

\section{Methods}

\section{Data acquisition and preprocessing}

The UCSC Xena browser (GDC Hub: https://gdc.xenahubs.net) was used to download CRC patients' data and transcriptome RNA sequence data from TCGA. The criteria of dataset selection were set as follows: (1) containing the patient's mRNA sequence data, and clinical information; and (2) containing the patient's prognostic information. A total of $222 \mathrm{CRC}$ samples were used in the subsequent analysis. A complete list of IRGs was obtained from the immunology database and analysis portal (ImmPort) database (https://immport.niaid.nih.gov). MSS-related genes were acquired from the human genome database (https://www.genecards.org/), and the genome could obtain complete information on a particular gene.

\section{Analysis of differentially expressed genes (DEGs)}

The change of gene expression was estimated using the $\mathrm{R}$ package with the empirical Bayesian method and a moderated t-test. DEGs were identified by R package LIMMA using the criterion (adjusted $P<0.05$ ) described in the R package. Benjamini-Hochberg correction was used to calculate the adjusted $P$ value of multiple tests to screen for DEGs between CRC and normal tissues. The intersection of DEGs, IRGs, and MSS-related genes (DE IR MSSGs) was used to further screen the differentially expressed MSS-mediated IRGs in CRC progression.

\section{Enrichment analysis of gene dataset}

The functional enrichment analysis was carried out, and the visualization of enrichment analysis was realized by using the $\mathrm{R}$ package "Clusterprofiler". Determined strict truncation values of the gene ontology (GO) and Kyoto Encyclopedia of Genes and Genomes (KEGG) pathway terms were $P<0.01$, and FDR (false discovery rate) $<0.05$.

\section{Development and verification of CRC immune-related markers}

Univariate Cox proportional hazards regression analysis was used to screen survival-related DE IR MSSGs in CRC patients. Survival-related DE IR MSSGs were considered to be $P<0.05$. The least absolute shrinkage and selector operation (LASSO) regression analysis was performed to determine DE IR MSSGs associated with survival, which were combined with expression profiles to minimize the overfine, and the R package "GLMnet" was used to find the optimal gene model. For the TCGA-COAD-READ cohort, the expression profile (standardized FPKM) was converted to TPM to obtain the risk score of each patient. 
The risk score $=$ gene 1 expression * coefficient + gene 2 expression * coefficient $+\ldots+$ gene $\mathrm{N}$ expression * coefficient $+b$. Each sample's risk score was calculated, and the best risk score's cut-off value was determined by using the $\mathrm{R}$ package. The patient was assigned to the high-risk group when the risk score was >cut-off value, otherwise, the patient was assigned to the low-risk group. The 12 DE IR MSSGs were stratified according to the difference in overall survival (OS) between the two risk groups as indicated by the Kaplan-Meier survival curve. The R package "Survival ROC" was used to calculate the area under the curve (AUC) to evaluate the TIME-dependent prognostic value of gene traits. When $P<0.05$ in the two-way logarithmic rank, it was of great significance to survival analysis. Multivariate Cox regression analysis was performed to verify whether these clinical features (including age, sex, clinical stage, histological grade, and TNM staging status) could predict the prognosis.

The correlation and differences between tumor-infiltrating immune cells and immune checkpoints in CRC populations

These immune cells, effector molecules, and immune checkpoints were identified by using the $\mathrm{R}$ software. The risk score of each patient was calculated, and their relationship with tumor-infiltrating immune cells (T cells, cytotoxic lymphocytes, B lineage, NK cells, monocytic lineage, myeloid dendritic cells, and neutrophils) and immune checkpoint molecules (PDCD1, CTLA4, HAVCR2, LAG3, CD274, and PDCD1LG2) was evaluated. The risk score of each sample was used to assess its relationship to these molecules.

\section{Mutation analysis}

The somatic mutation data (SNP and small INDEL, MuTect2 mutation aggregation and masking) of STAD patients were obtained from the TCGA database (https://gdc.xenahubs.net/download/TCGACOAD-READ/Xena_Matrices/TCGA-COAD-READ.mutect2_snv.tsv .gz). The R software was used to select the gene mutation data from 12 DE IR MSSGs to determine the difference between the two groups of CRC patients.

\section{Statistical analysis}

Graphpad 8, SPSS and R software were used to analyze the corresponding data. The MSS score was evenly divided into low and high groups using the $\mathrm{R}$ package MaxStat to reduce batch calculation. The critical genes in differential gene analysis were obtained by transforming the P-value into FDR using the Benjamini-Hochberg method. The volcano map, heat map, and bubble map were generated using the $\mathrm{R}$ packages "ggplot2", "pheatmap", and "clusterProfiler", respectively. The differences between variables were analyzed by t-test, non-parametric test, and chi-square test. The prognostic ability of MSS-mediated immune-related risk characteristics and clinical features was evaluated by univariate, LASSO, and multivariate Cox regression analysis.

\section{Abbreviations}




\begin{tabular}{|ll|}
\hline English abbreviations & English full name \\
\hline CRC & colorectal cance \\
\hline MSS & microsatellite stability \\
\hline LASSO & least absolute shrinkage and selector operation \\
\hline 12 DE IRGs & 12 differentially expressed immune-related genes \\
\hline ICB & immune checkpoint inhibitors \\
\hline MSI & Microsatellite instability \\
\hline MMR & mismatch repair \\
\hline TIME & immune microenvironment \\
\hline TCGA & The Cancer Genome Atlas \\
\hline
\end{tabular}

\section{Declarations}

\section{Acknowledgments}

Funding: The present study was supported by the National Natural Science Foundation of China (31800745), Changzhou High-Level Medical Talents Training Project (No. 2016CZBJ001), High-Level Talents Project of Jiangsu Commission of Health (LGY2020034), and the Young Talent Development Plan of Changzhou Health Commission (CZQM2020044).

Contributions: (I) Conception and design: Y Li, X Zheng, LJ Chen; (II) Administrative support: X Zheng, LJ Chen; (III) Collection and assembly of data: Y Li, H Huang, J Feng, YL Zhu; (IV) Data analysis and interpretation: Y Li, TW Jiang, B Xu, X Zheng, LJ Chen; (V) Manuscript writing: All authors; (VI) Final approval of manuscript: All authors. \#These authors contributed equally to this work.

Conflicts of Interest: The authors have no conflicts of interest to declare.

Ethical Statement: The authors are accountable for all aspects of the work in ensuring that questions related to the accuracy or integrity of any part of the work are appropriately investigated and resolved.

Consent for publication: All authors consent to the publication of the article in this journal.

Availability of data and materials: All data and materials are available.

\section{References}

1. Testa U, Pelosi E, Castelli G. Colorectal cancer: genetic abnormalities, tumor progression, tumor heterogeneity, clonal evolution and tumor-initiating cells. Med Sci (Basel) 2018;6. 
2. De Rosa M, Pace U, Rega D, et al. Genetics, diagnosis and management of colorectal cancer (Review). Oncol Rep 2015;34:1087-96.

3. Haraldsdottir S, Einarsdottir HM, Smaradottir A, et al. [Colorectal cancer - review]. Laeknabladid 2014;100:75-82.

4. Horn L, Spigel DR, Vokes EE, et al. Nivolumab Versus Docetaxel in Previously Treated Patients With Advanced Non-Small-Cell Lung Cancer: Two-Year Outcomes From Two Randomized, Open-Label, Phase III Trials (CheckMate 017 and CheckMate 057). J Clin Oncol 2017;35:3924-33.

5. Eggermont AMM, Crittenden M, Wargo J. Combination Immunotherapy Development in Melanoma. Am Soc Clin Oncol Educ Book 2018;38:197-207.

6. Overman MJ, McDermott R, Leach $\mathrm{JL}$, et al. Nivolumab in patients with metastatic DNA mismatch repair-deficient or microsatellite instability-high colorectal cancer (CheckMate 142): an open-label, multicentre, phase 2 study. Lancet Oncol 2017;18:1182-91.

7. Evrard C, Tachon G, Randrian V, et al. Microsatellite Instability: Diagnosis, Heterogeneity, Discordance, and Clinical Impact in Colorectal Cancer. Cancers (Basel) 2019;11.

8. Jiricny J. The multifaceted mismatch-repair system. Nat Rev Mol Cell Biol 2006;7:335-46.

9. Ganesh K, Stadler ZK, Cercek A, et al. Immunotherapy in colorectal cancer: rationale, challenges and potential. Nat Rev Gastroenterol Hepatol 2019;16:361-75.

10. Llosa NJ, Cruise M, Tam A, et al. The vigorous immune microenvironment of microsatellite instable colon cancer is balanced by multiple counter-inhibitory checkpoints. Cancer Discov 2015;5:43-51.

11. Rodriguez-Soler M, Perez-Carbonell L, Guarinos C, et al. Risk of cancer in cases of suspected lynch syndrome without germline mutation. Gastroenterology 2013;144:926-32 e1; quiz e13-4.

12. Le DT, Uram JN, Wang H, et al. PD-1 Blockade in Tumors with Mismatch-Repair Deficiency. N Engl J Med 2015;372:2509-20.

13. Sillo TO, Beggs AD, Morton DG, et al. Mechanisms of immunogenicity in colorectal cancer. Br J Surg 2019;106:1283-97.

14. Boland CR, Goel A. Microsatellite instability in colorectal cancer. Gastroenterology 2010;138:2073-87 e3.

15. Song Q, Shang J, Yang Z, et al. Identification of an immune signature predicting prognosis risk of patients in lung adenocarcinoma. J Transl Med 2019;17:70.

16. Shen S, Wang G, Zhang R, et al. Development and validation of an immune gene-set based Prognostic signature in ovarian cancer. EBioMedicine 2019;40:318-26. 
17. Wu J, Zhao Y, Zhang J, et al. Development and validation of an immune-related gene pairs signature in colorectal cancer. Oncoimmunology 2019;8:1596715.

18. Lech G, Slotwinski R, Slodkowski M, et al. Colorectal cancer tumour markers and biomarkers: Recent therapeutic advances. World J Gastroenterol 2016;22:1745-55.

19. Rizvi S, Wang J, El-Khoueiry AB. Liver Cancer Immunity. Hepatology 2020.

20. Guinney J, Dienstmann R, Wang X, et al. The consensus molecular subtypes of colorectal cancer. Nat Med 2015;21:1350-6.

21. Akbari V, Kallhor M, Mollashahi B, et al. Transcriptome and Network Dissection of Microsatellite Stable and Highly Instable Colorectal Cancer. Asian Pac J Cancer Prev 2019;20:2445-54.

22. Yu L, Yang $X, X u C$, et al. Comprehensive analysis of the expression and prognostic value of $C X C$ chemokines in colorectal cancer. Int Immunopharmacol 2020;89:107077.

23. Sun L, Zhang Y, Zhang C. Distinct Expression and Prognostic Value of MS4A in Gastric Cancer. Open Med (Wars) 2018;13:178-88.

24. Guo Y, Duan Z, Jia Y, et al. HER4 isoform CYT2 and its ligand NRG1III are expressed at high levels in human colorectal cancer. Oncol Lett 2018;15:6629-35.

25. Srabovici N, Mujagic Z, Mujanovic-Mustedanagic J, et al. Interleukin 13 expression in the primary breast cancer tumour tissue. Biochem Med (Zagreb) 2011;21:131-8.

26. Aziz S, Ahmed SS, Ali A, et al. Salivary Immunosuppressive Cytokines IL-10 and IL-13 Are Significantly Elevated in Oral Squamous Cell Carcinoma Patients. Cancer Invest 2015;33:318-28.

27. Formentini A, Braun P, Fricke $H$, et al. Expression of interleukin-4 and interleukin-13 and their receptors in colorectal cancer. Int J Colorectal Dis 2012;27:1369-76.

28. Zhang Y, Zhao X, Deng L, et al. High expression of FABP4 and FABP6 in patients with colorectal cancer. World J Surg Oncol 2019;17:171.

29. Wang X, Prager BC, Wu Q, et al. Reciprocal Signaling between Glioblastoma Stem Cells and Differentiated Tumor Cells Promotes Malignant Progression. Cell Stem Cell 2018;22:514-28 e5.

30. Patel O, Clyde D, Chang M, et al. Pro-GRP-derived peptides are expressed in colorectal cancer cells and tumors and are biologically active in vivo. Endocrinology 2012;153:1082-92.

31. Khurram SA, Whawell SA, Bingle L, et al. Functional expression of the chemokine receptor XCR1 on oral epithelial cells. J Pathol 2010;221:153-63. 
32. Li B, Cai S, Zhao Y, et al. Nerve growth factor modulates the tumor cells migration in ovarian cancer through the WNT/beta-catenin pathway. Oncotarget 2016;7:81026-48.

33. Fang JB, Wang L. [The function of tumor specific antigen (MAGE) in tumor immunotherapy]. Sheng Li Ke Xue Jin Zhan 2005;36:273-5.

34. Xiao J, Chen HS. Biological functions of melanoma-associated antigens. World J Gastroenterol 2004;10:1849-53.

35. Xiao J, Chen HS. [Biological functions of melanoma-associated antigens (MAGEs) in cell activities]. Ai Zheng 2005;24:124-8.

36. Sudo T, Kuramoto T, Komiya S, et al. Expression of MAGE genes in osteosarcoma. J Orthop Res 1997;15:128-32.

37. van der Bruggen $\mathrm{P}$, Traversari C, Chomez P, et al. A gene encoding an antigen recognized by cytolytic $T$ lymphocytes on a human melanoma. J Immunol 2007;178:2617-21.

38. Fang Y, Shen ZY, Zhan YZ, et al. CD36 inhibits beta-catenin/c-myc-mediated glycolysis through ubiquitination of GPC4 to repress colorectal tumorigenesis. Nat Commun 2019;10:3981.

\section{Tables}

Table 1 Gene list and immune category of 12 DE IR MSSGs signature

\begin{tabular}{llll} 
Gene & GC ID & Name & Category \\
\hline CXCL1 & GC04P073869 & C-X-C Motif Chemokine Ligand 1 & Protein Coding \\
\hline CD36 & GC07P080369 & CD36 Molecule & Protein Coding \\
\hline FABP4 & GC08M081478 & Fatty Acid Binding Protein 4 & Protein Coding \\
\hline MS4A2 & GC11P060088 & Membrane Spanning 4-Domains A2 & Protein Coding \\
\hline NRG1 & GC08P031639 & Neuregulin 1 & Protein Coding \\
\hline VGF & GC07M101162 & VGF Nerve Growth Factor Inducible & Protein Coding \\
\hline GRP & GC18P059220 & Gastrin Releasing Peptide & Protein Coding \\
\hline HDC & GC15M050241 & Histidine Decarboxylase & Protein Coding \\
\hline XCL1 & GC01P168576 & X-C Motif Chemokine Ligand 1 & Protein Coding \\
\hline NGF & GC01M115285 & Nerve Growth Factor & Protein Coding \\
\hline MAGEA1 & GC0XP153179 & MAGE Family Member A1 & Protein Coding \\
\hline IL13 & GC05P132656 & Interleukin 13 & Protein Coding
\end{tabular}


Table 2. Relationship between risk score and clinicopathological features in CRC patients 


\begin{tabular}{|c|c|c|c|c|c|}
\hline \multirow[t]{2}{*}{ Clinical characteristics } & \multirow[t]{2}{*}{ Cases } & \multicolumn{2}{|c|}{ Risk group } & \multirow[t]{2}{*}{$x^{2}$} & \multirow[t]{2}{*}{$P$ value } \\
\hline & & Low & High & & \\
\hline Gender & & & & 6.8360 & $0.0089 *$ \\
\hline Male & 100 & 67 & 33 & & \\
\hline Female & 108 & 53 & 55 & & \\
\hline Age & & & & 0.3180 & 0.5639 \\
\hline$\leq 65$ years old & 90 & 47 & 43 & & \\
\hline$>65$ years old & 132 & 74 & 58 & & \\
\hline Clinical stage & & & & 1.7370 & 0.7840 \\
\hline प & 36 & 23 & 13 & & \\
\hline प & 83 & 43 & 40 & & \\
\hline ૫ & 65 & 34 & 31 & & \\
\hline IV & 32 & 18 & 14 & & \\
\hline NA & 6 & 3 & 3 & & \\
\hline T stage & & & & 1.9440 & 0.7460 \\
\hline T1 & 4 & 2 & 2 & & \\
\hline $\mathrm{T} 2$ & 38 & 22 & 16 & & \\
\hline T3 & 147 & 76 & 71 & & \\
\hline T4 & 31 & 20 & 11 & & \\
\hline Tx & 2 & 1 & 1 & & \\
\hline $\mathrm{N}$ stage & & & & 0.9779 & 0.8066 \\
\hline NO & 124 & 68 & 56 & & \\
\hline N1 & 51 & 30 & 21 & & \\
\hline $\mathrm{N} 2$ & 45 & 22 & 23 & & \\
\hline $\mathrm{Nx}$ & 2 & 1 & 1 & & \\
\hline M stage & & & & 0.8065 & 0.6681 \\
\hline MO & 161 & 89 & 72 & & \\
\hline M1 & 32 & 18 & 14 & & \\
\hline$M x$ & 26 & 12 & 14 & & \\
\hline
\end{tabular}


Bold signifies $P<0.05$; CRC, colorectal cancer.

Table 3. Multivariate Cox regression analysis of overall survival in CRC patients

\begin{tabular}{|c|c|c|}
\hline \multirow[t]{2}{*}{ Clinical parameters } & \multicolumn{2}{|l|}{ Multi-variate } \\
\hline & $H R(95 \% C l)$ & $P$ value \\
\hline \multirow[t]{2}{*}{ Risk group (low:high) } & 7.5573 & $<0.001 *$ \\
\hline & (3.2931-17.3428) & \\
\hline \multirow[t]{2}{*}{ Gender(female:male) } & 0.5033 & $0.0376 *$ \\
\hline & $(0.2634-0.9696)$ & \\
\hline \multirow[t]{2}{*}{ Age(years) $(\leq 66: \llbracket 66)$} & 2.6688 & $0.0066^{*}$ \\
\hline & $(1.3139-5.4208)$ & \\
\hline \multirow[t]{2}{*}{ 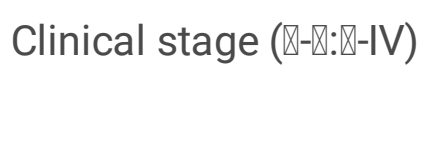 } & 6.4454 & $0.0391 *$ \\
\hline & $(1.0968-37.8756)$ & \\
\hline \multirow[t]{2}{*}{ T stage (T1-T2:T3-T4) } & 0.8319 & 0.6620 \\
\hline & $(0.3644-1.8990)$ & \\
\hline \multirow[t]{2}{*}{ N stage (N0:N1-N2) } & 0.3707 & 0.2228 \\
\hline & $(0.0751-1.8283)$ & \\
\hline \multirow[t]{2}{*}{ M stage (M0:M1) } & 6.4454 & 0.6491 \\
\hline & $(1.0968-37.8756)$ & \\
\hline
\end{tabular}

Bold signifies $P<0.05$; CRC, colorectal cancer.

\section{Figures}




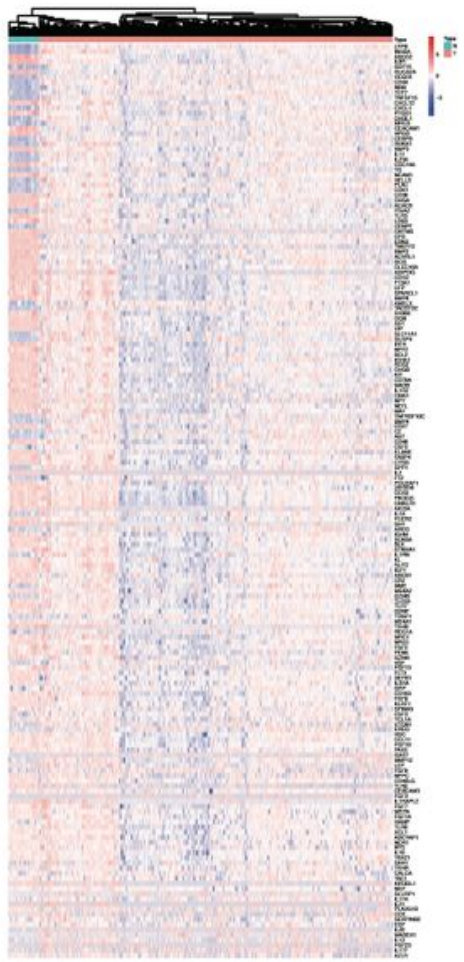

\section{Figure 1}

We analyzed the difference and obtained the DEGs by heat map and volcano map 

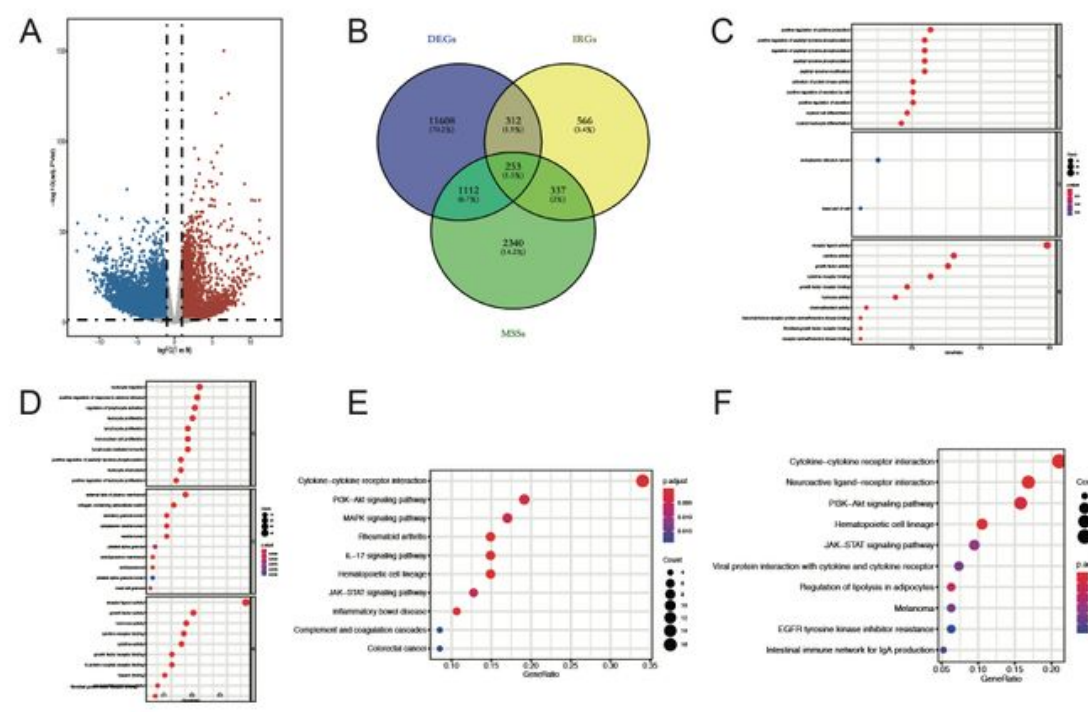

F

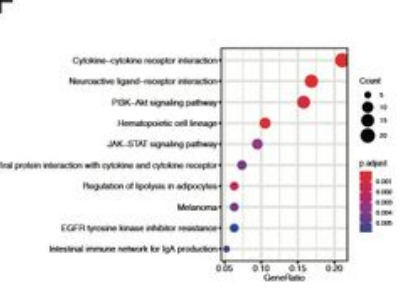

\section{Figure 2}

The intersection of MSS-related genes, IRGs, and DEGs in CRC identified 253 differentially expressed IRGs (DE IR MSSGs) 


\begin{tabular}{|c|c|c|c|c|c|}
\hline$\overline{\text { Gene }}$ & Coefficient & & & Pvalue & Hazard Ratio $(95 \% \mathrm{Cl})$ \\
\hline$\overline{\mathrm{CXCL} 1}$ & -0.143 & 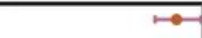 & & 0.036 & $0.867(0.759-0.99)$ \\
\hline $\mathrm{CD} 36$ & 0.202 & & $\longmapsto$ & 0.04 & $1.224(1.009-1.484)$ \\
\hline FABP4 & 0.136 & & $\mapsto$ & 0.008 & $1.145(1.036-1.266)$ \\
\hline MS4A2 & -0.437 & $\longmapsto$ & & 0.021 & $0.646(0.445-0.937)$ \\
\hline NRG1 & -0.595 & $\longmapsto$ & & 0.014 & $0.551(0.342-0.888)$ \\
\hline VGF & 0.149 & & $\longrightarrow$ & 0.036 & $1.16(1.01-1.333)$ \\
\hline GRP & 0.176 & & $\longmapsto$ & 0.018 & $1.192(1.031-1.379)$ \\
\hline HDC & -0.443 & $\longmapsto$ & & 0.021 & $0.642(0.44-0.935)$ \\
\hline $\mathrm{XCL} 1$ & 0.362 & & $\longmapsto$ & 0.004 & $1.436(1.121-1.839)$ \\
\hline NGF & 0.5 & & $\longmapsto$ & 0.002 & $1.648(1.21-2.246)$ \\
\hline MAGEA1 & 0.285 & & $\longmapsto$ & $P<0.001$ & $1.329(1.167-1.514)$ \\
\hline IL13 & -1.485 & $\longmapsto$ & & 0.037 & $0.226(0.056-0.917)$ \\
\hline
\end{tabular}

\section{Figure 3}

Using profiles of DE IR MSSGs associated with OS, we performed LASSO regression analysis and identified 12 CRC-specific prognostic DE IR MSSGs 


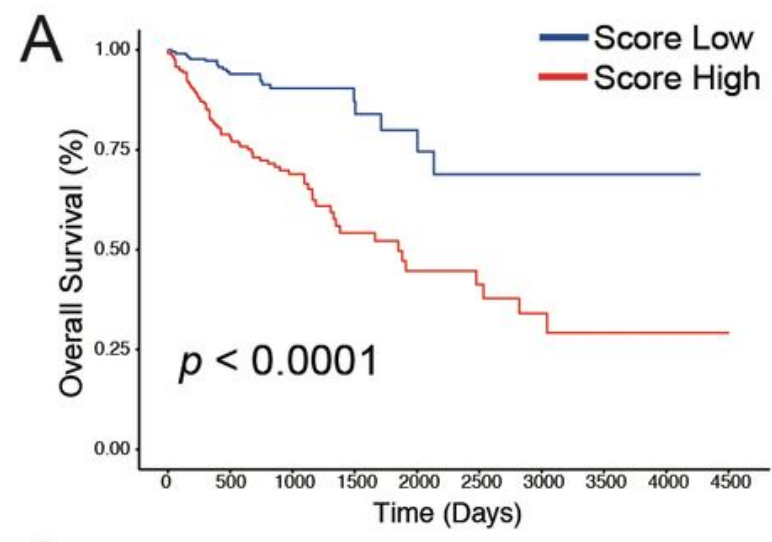

C
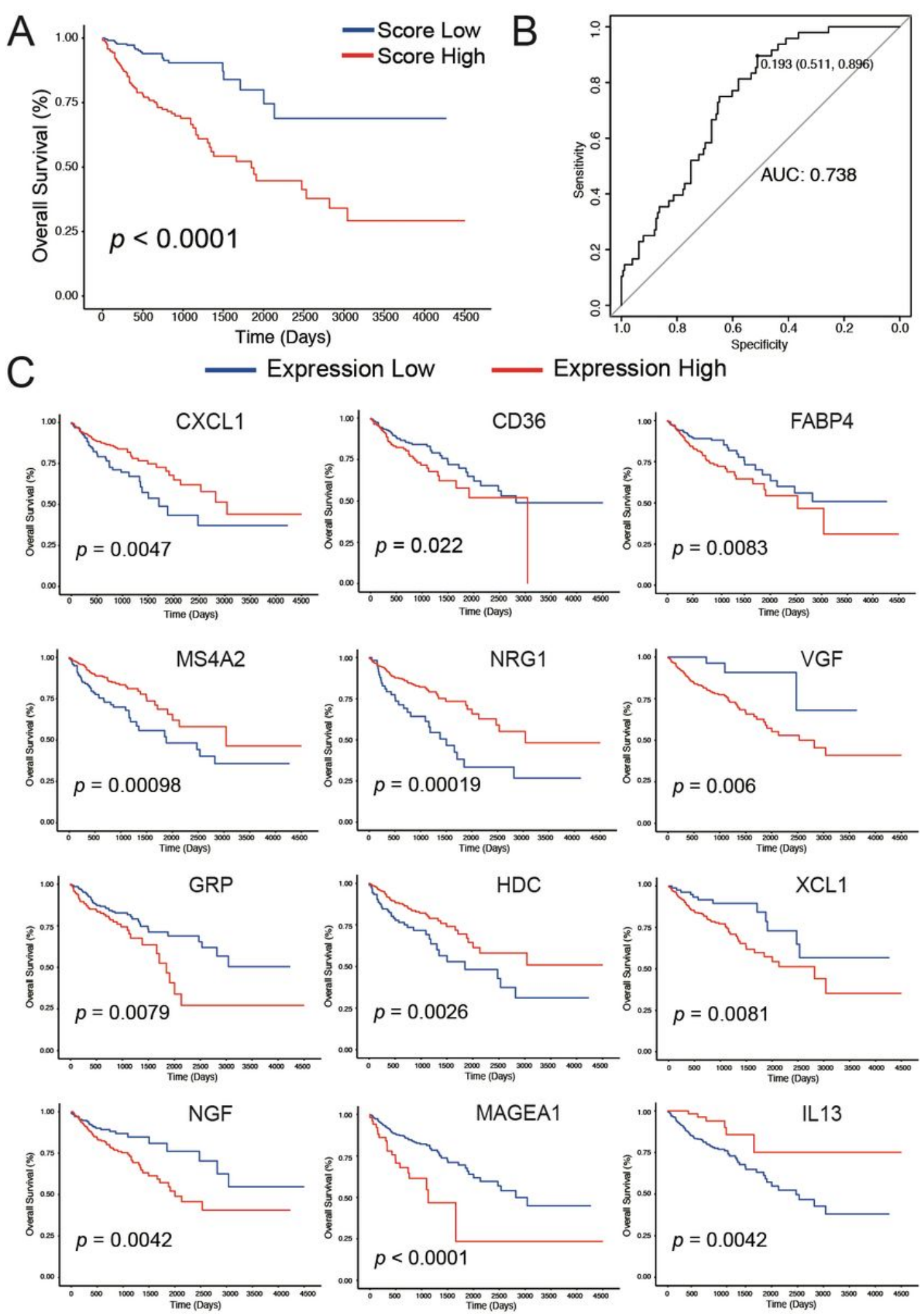

\section{Figure 4}

By analyzing the relationship between 12 DE IR MSSGs and OS, we found that OS was significantly better in the low-risk group 

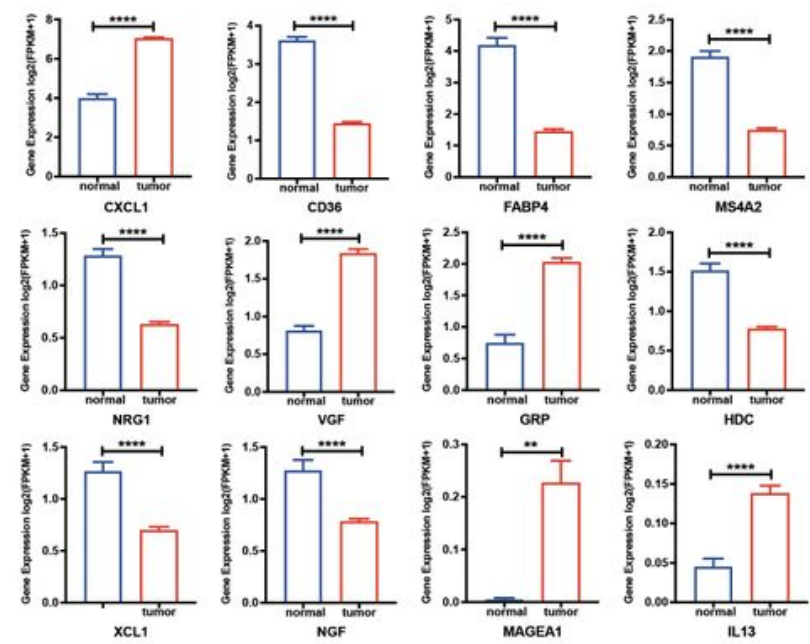

Figure 5

The expressions of CXCL1, VGF, GRP, MAGEA1, and IL13 in CRC patients were significantly up-regulated compared with the normal controls, while the expressions of CD36, FABP4, MS4A2, NRG1, HDC, XCL1, and NGF were significantly down-regulated 


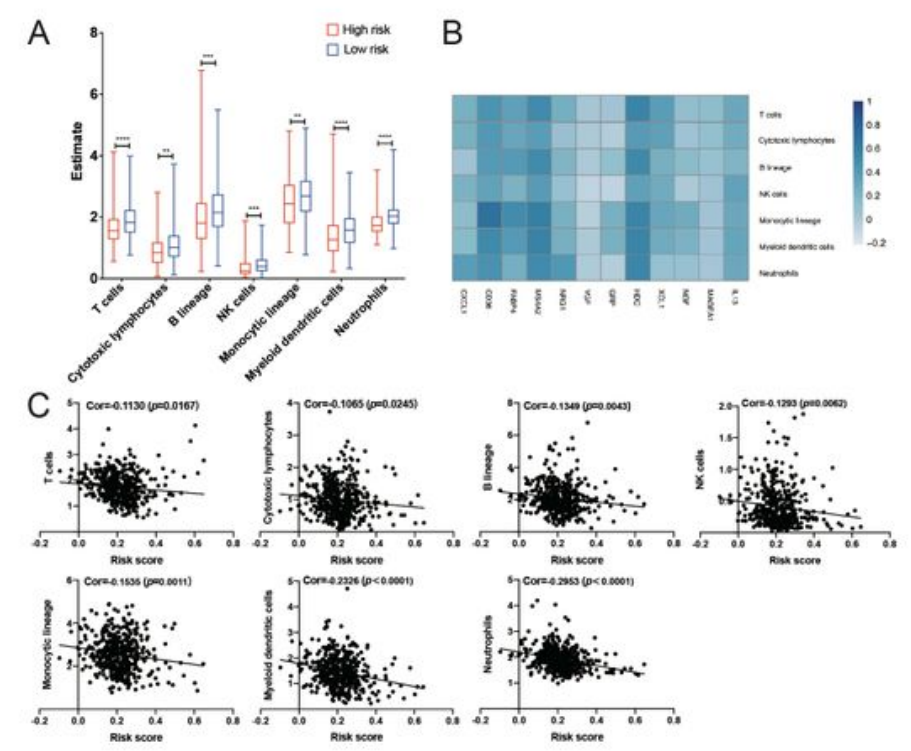

\section{Figure 6}

The results showed that the infiltration of T cells, cytotoxic lymphocytes, B lineage, NK cells, monocytic lineage, myeloid dendritic cells, and neutrophils was higher in the low-risk group, which might be related to a better anti-tumor effect 


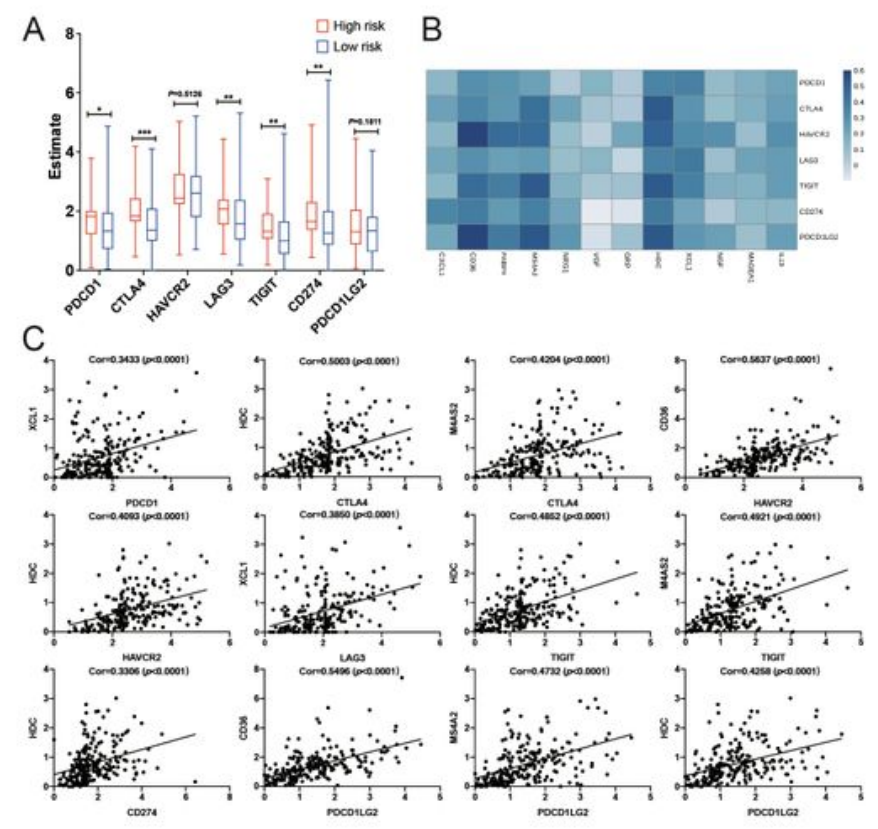

Figure 7

In the high-risk group, the expressions of PDCD1, CTLA4, HAVCR2, LAG3, TIGIT, CD274, and PDCDLG2 were up-regulated 

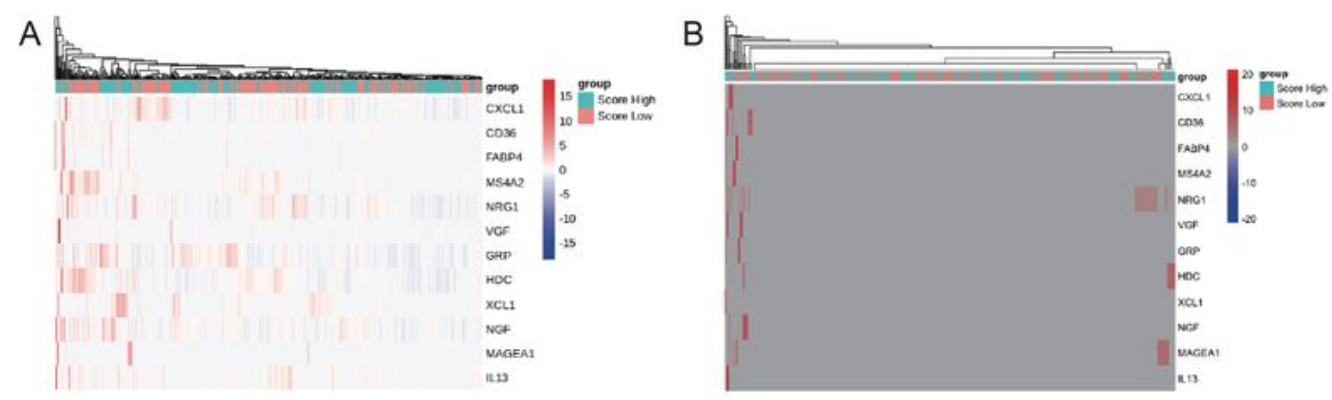

\section{Figure 8}

By analyzing the relationship between the gene expression and mutation of the two groups, we found that there was no significant difference in gene mutation 